\title{
Drift-sound and drift-Alfvén eigenmodes in toroidal plasmas
}

\author{
Ya. I. Kolesnichenko ${ }^{1}$, V. V. Lutsenko ${ }^{1}$, A. WelleR ${ }^{2}$, H. Thomsen ${ }^{2}$, Yu. V. Yakovenko ${ }^{1}$, J. Geiger ${ }^{2}$ and \\ A. WERNER ${ }^{2}$ \\ 1 Institute for Nuclear Research, National Academy of Sciences of Ukraine - Prospekt Nauky 47, Kyiv 03680, Ukraine \\ 2 Max-Planck-Institut für Plasmaphysik, IPP-EURATOM Association - D-17491 Greifswald, Germany
}

PACS $52.30 . \mathrm{Ex}$ - Two-fluid and multi-fluid plasmas
PACS $52.35 .-\mathrm{g}$ - Waves, oscillations, and instabilities in plasmas and intense beams
PACS $52.55 . \mathrm{Fa}$ - Tokamaks, spherical tokamaks
PACS $52.55 . \mathrm{HC}$ - Stellarators, spheromaks, compact tori, bumpy tori, and other toroidal confine-
$\quad$ ment devices

\begin{abstract}
Equations describing eigenmodes with the frequencies of the order of the geodesic acoustic frequency and the electron/ion diamagnetic frequency in toroidal plasmas are derived and analyzed, a code solving them is developed. It is shown that there exist drift-sound eigenmodes and a new kind of drift-Alfvén eigenmodes. This is done by means of an analytical consideration and numerical modelling of particular discharges in the stellarator Wendelstein 7-AS.
\end{abstract}

Introduction. - Recently, instabilities with frequencies below that of the Toroidicity-induced Alfvén Eigenmodes (TAE) attracted considerable attention in fusion research. These Low Frequency (LF) instabilities may strongly deteriorate the confinement of fast ions [1]; they are not necessarily harmful, in which case they can be used for plasma diagnostics [2,3]. LF instabilities occur in all types of toroidal plasma systems. In particular, Reversed Shear Alfvén Eigenmodes (RSAE) or Alfvén Cascades (AC) were observed in JET and extensively studied theoretically $[4,5]$; Non-conventional Global Alfvén Eigenmodes (NGAE) were predicted to exist in stellarators and seem to be observed in Wendelstein 7-AS (W7-AS) [3,6]; Beta-induced Alfvén Acoustic Eigenmodes (BAAE) were observed in the NSTX spherical torus [7], the Sound Cascades (SC) were observed in ASDEX-Upgrade [8]. Despite efforts of many theorists, a number of features of experimentally observed LF instabilities remain a mystery. In particular, it is not clear how the BAAE modes, which are actually sound waves and, thus, are strongly damped in isothermic plasmas, can account for experiments described in ref. [7]; why a LF instability observed in DIII-D manifests itself even at any beam power [9]. Furthermore, there are different interpretations of Beta-induced Alfvén Eigenmodes (BAE) observed in DIII-D many years ago [1]. Both the BAAE in NSTX and the mentioned instabilities in DIII-D have the frequencies below the frequency of the Geodesic Acoustic Mode (GAM), $\omega_{G}[10]$. The modes with the frequencies below/about $\omega_{G}$, which we may refer to as sub-GAM modes, were observed also in W7-AS [11]. The nature of some of them remains unclear. The purpose of this Letter is to show a possible way to understanding the mentioned mysterious experimental facts; as a specific example, sub-GAM instabilities observed in W7-AS are considered.

Our basic idea is that plasma compressibility and finite diamagnetic frequencies of the electrons and ions, $\omega_{* e}$ and $\omega_{* i}$, play an important role in LF instabilities. Taking the diamagnetic frequencies into account may immediately explain why sound perturbations are weakly damped even in isothermic plasmas: due to finite $\omega_{*}$, the frequency of sound perturbations does not go to zero when $k_{\|} \rightarrow 0$ ( $k_{\|}$is the longitudinal wave number), which implies that $\omega / k_{\|} \gg v_{\mathrm{th}, i}\left(\omega\right.$ is the wave frequency, $v_{\mathrm{th}, i}$ is the ion thermal velocity) when $k_{\|}$is sufficiently small. Moreover, finite $\omega_{*}$ breaks the symmetry of the dispersion relation with respect to the sign of $\omega$, which, in particular, may lead to the existence of new modes. It follows from the foregoing that the ideal MHD approximation may be insufficient for the description of LF modes. Note that this was realized long time ago; nevertheless, typically one usually uses ideal MHD to describe destabilized eigenmodes. On the other hand, studies beyond ideal MHD showed new interesting results: Kinetic Ballooning Modes (KBM) and Energetic Particle Modes (EPM) of drift type were predicted [12], and new features of LF instabilities were 
revealed due to taking into account plasma compressibility and the ion diamagnetic frequency on the same footing [13]. However, the mentioned results are relevant only to perturbations rotating in the ion diamagnetic direction (these perturbations belong to Alfvén branches, see fig. 1 and its description in the next section); another important class of perturbation - sound perturbations rotating in the electron diamagnetic direction - is not considered yet (although the basic equations of ref. [13] could be used to study sound modes). Moreover, the modes in refs. [12,13] are studied in the framework of the ballooning formalism [14], which assumes translational invariance of radial mode structures. This invariance implies connection of the modes with rational- $\iota$ flux surfaces [for example, with $\iota=n / m$ for the ballooning modes, with $\iota=2 n /(2 m-1)$ for the Toroidicity-induced Alfvén Eigenmodes (TAE) etc.; here $\iota$ is the rotational transform, $m$ and $n$ are the poloidal and toroidal mode numbers, respectively]. Therefore, the ballooning formalism in its standard form cannot describe the modes with frequencies close to extrema of continua away from the rational surfaces [like Global Alfvén Eigenmodes (GAE), NGAE and RSAE], where the translational symmetry breaks down (note that these modes can be treated by Fourier transformation of the mode equations in the radial variable in the vicinity of a continuum extremum [15], which can be considered as a generalization of the ballooning approach [16]). This motivated us to derive new equations describing both drift-Alfvén eigenmodes and drift-sound eigenmodes in the real space, without using the ballooning ansatz, which are valid for both tokamaks and stellarators.

A difficulty to be overcome for describing the eigenmodes of the sound type is that the equation for the perturbations of the drift-sound type does not contain radial derivative terms and, thus, determine only continua but not eigenmodes. We resolve this problem by taking into account the magnetic field inhomogeneity, which couples the drift-sound perturbations with the drift-Alfvén perturbations.

Basic equations and the code BOAS. - We proceed from the collisionless fluid equations derived in refs. $[17,18]$, which take into account the anisotropy of the plasma pressure and the gyroviscous cancellation [19]. We assume that $v_{\text {th }, i} \ll \omega / k_{\|} \ll v_{\text {th }, e}$, where $v_{\text {th,e }}$ is the electron thermal velocity. In this case the longitudinal thermal flux dominates the electron energy balance; therefore, the electron temperature is flattened out along the field lines: $\mathbf{B} \cdot \nabla T_{e}=0$ (with $\mathbf{B}$ the magnetic field strength and $T_{e}$ the electron temperature), which implies that the electron temperature remains isotropic in the perturbed state. In addition to this equation, we use the equation of motion (without the inertia term) and the quasi-neutrality condition $n_{e}=n_{i}$ (where $n_{e / i}$ is the electron/ion density) to describe electrons. For the ion component, we neglect the longitudinal thermal fluxes due to the condition $\omega / k_{\|} \gg v_{\text {th }, i}$ (this implies that there is no mecha- nism to maintain the pressure isotropy and, thus, the perturbed ion pressure is strongly anisotropic). To eliminate fast magnetoacoustic waves from the consideration, we assume that the total perpendicular pressure of the magnetic field and plasma is not disturbed. For low- $\beta$ plasmas, the plasma pressure can be neglected, which leads to the condition $\tilde{B}_{\|} \approx 0$ (tilde labels perturbations). Finally, we take the equilibrium magnetic field, $B_{0}$, and perturbed quantities, $\tilde{X}$, in the forms:

$$
\begin{gathered}
B_{0}=\bar{B}\left(1+\frac{1}{2} \sum_{\mu \nu} \epsilon_{B}^{(\mu \nu)}(r) e^{i \mu \theta-i \nu N \phi}\right) \\
\tilde{X}=\sum_{m, n} \tilde{X}_{m, n}(r) e^{i m \theta-i n \phi-i \omega t}
\end{gathered}
$$

where $r, \theta$, and $\phi$ are Boozer coordinates, $\epsilon_{B}^{(-\mu,-\nu)}=\epsilon_{B}^{(\mu \nu)}$, $N$ is the number of the field periods. Then we obtain the following set of equations (details will be published elsewhere):

$$
\text { see eqs. (3) and (4) }
$$

where $\Phi$ is the perturbed scalar potential of the electromagnetic field, $\zeta_{m n} \equiv i \omega \nabla_{\|} \tilde{v}_{\| m n}$, with $\tilde{v}_{\|}$the longitudinal perturbed velocity of the ion component, $\tau=T_{i} / T_{e}, T_{e / i}$ is the electron/ion temperature, $k_{m n}=(m \iota-n) / R$ is the longitudinal wave number, $R$ is the major radius of the torus, $c_{e i}^{2}=T_{e} / M_{i}, M_{i}$ the ion mass, $\omega_{* i}=m c p_{i}^{\prime} /\left(e_{i} B n_{i} r\right)$ and $\omega_{* e}=m c T_{e} n_{e}^{\prime} /\left(e_{e} B n_{e} r\right)$, with $p_{i}$ the ion pressure, prime denotes the radial derivative, $\nu_{\iota}$ is the fraction of the rotational transform produced by the plasma current ( $\nu_{\iota}=1$ in tokamaks, $\nu_{\iota}=0$ in currentless stellarators), $\delta_{0} \gtrsim 1$ characterizes the plasma shape [3],

$$
\omega_{G}=\frac{c_{e i}}{R} \frac{\epsilon_{t}}{\epsilon} \sqrt{\frac{2}{\delta_{0}}\left(1+\frac{7}{4} \tau\right)},
$$

$\epsilon=r / R, \epsilon_{t}=\epsilon_{B}^{(1,0)}$. In these equations, $\Phi$ describes Alfvénic perturbations, whereas $\zeta$ describes perturbations of the sound type. The magnitudes $\Phi$ and $\zeta$ are coupled due to finite $\epsilon_{B}^{(\mu \nu)}$. For simplicity, the coupling terms were derived in assumptions of homogeneous plasma temperature and high mode numbers; in addition, we took $\delta_{0}(r)=$ const and $\nu_{\iota}(r)=$ const. Equation (6) is written with taking into account that the harmonics with high $\mu$, $\nu$ weakly contribute to $\omega_{G}$ (see ref. [3]); in addition, the harmonics of $B_{0}$ with $\mu>1$ are neglected (therefore, $\omega_{G}$ is somewhat underestimated).

In order to solve the coupled drift-sound and driftAlfvén equations, a numerical code BOAS (Branches Of Alfvén and Sound modes) was developed. The code calculates both the continuum and discrete modes. It can also allow for finite ion Larmor radius (FLR) effects when the left-hand side of eq. (4) is supplemented by a fourthderivative term, $\alpha r d^{4} \Phi_{m+\mu, n+\nu N} / d r^{4}$, where $\alpha$ is proportional to square of the ion Larmor radius (see ref. [20] for the case of $\omega>\omega_{*}$ and $\omega>c_{e i} / R$; a more general expression for $\alpha$ can be found in ref. [21]). 


$$
\begin{aligned}
& \omega^{2}\left(\omega-\omega_{* e}\right) \zeta_{m n}-k_{m n}^{2} c_{e i}^{2}\left[\omega-\omega_{* i}+3 \tau\left(\omega-\omega_{* e}\right)\right] \zeta_{m n} \\
& =-\frac{c}{\bar{B}} c_{e i}^{2}\left[\omega(1+2 \tau)+\omega_{* i}\right]\left(\omega-\omega_{* i}\right) \frac{k_{m, n}^{2}}{r}\left[\mu \epsilon_{B}^{(\mu \nu)} \frac{d \Phi_{m+\mu, n+\nu N}}{d r}+(m+\mu) \frac{d \epsilon_{B}^{(\mu \nu)}}{d r} \Phi_{m+\mu, n+\nu N}\right], \\
& \quad \frac{1}{r} \frac{d}{d r} r\left[\frac{\omega^{2}-\omega_{G}^{2}}{v_{A}^{2}}\left(1-\frac{\omega_{* i}}{\omega}\right)-k_{m+\mu, n+\nu N}^{2}\right] \frac{d \Phi_{m+\mu, n+\nu N}}{d r} \\
& \quad-\left\{\frac{(m+\mu)^{2}}{r^{2}}\left[\frac{\omega^{2}-\omega_{G}^{2}}{v_{A}^{2}}\left(1-\frac{\omega_{* i}}{\omega}\right)-k_{m+\mu, n+\nu N}^{2}\right]+\frac{k_{m+\mu, n+\nu N}}{r}\left[\left(r k_{m+\mu, n+\nu N}^{\prime}\right)^{\prime}\right.\right. \\
& \left.\left.\quad-\frac{m+\mu}{R}\left(3 \iota^{\prime}+r \iota^{\prime \prime}\right) \nu_{\iota}\right]\right\} \Phi_{m+\mu, n+\nu N} \\
& =\frac{\bar{B}}{c r \omega \delta_{0}}\left[\frac{c_{e i}^{2}}{v_{A}^{2}}(1+2 \tau) \frac{d \epsilon_{B}^{(\mu \nu)}}{d r}(m+\mu) \zeta_{m n}-\frac{d}{d r} \frac{c_{e i}^{2}}{v_{A}^{2}}(1+2 \tau) \mu \epsilon_{B}^{(\mu \nu)} \zeta_{m n}\right]
\end{aligned}
$$

Drift-sound eigenmodes, drift-Alfvén eigenmodes, and their kinetic counterparts. - Let us analyze these equations. First of all, we consider the continuum, assuming that the coupling terms weakly disturb it. The continuum branches depend on the ratio of $\omega_{G} / \omega_{* i}$. A sketch of them for $\omega_{G}>\omega_{* i}$ is shown in fig. 1. Note that we took $\omega>0$ for the modes rotating in the ion diamagnetic direction and $\omega<0$ for the modes rotating in the electron direction, in which case $m<0$ and $n<0$. We observe that there are Alfvén branches with both $\omega>0$ and $\omega<0$, but when $\omega<\omega_{* i}$, the Alfvén branch is positive. The sound continuum branch near which weakly damped modes are possible is negative. Similar conclusions can be drawn when $\omega_{G}<\omega_{* i}$ and $\omega_{G}<\left|\omega_{* e}\right|$ despite different behaviour of the continuum branches.

An analysis of several different W7-AS discharges (\#39029, \#43348, \#54022 and others) confirmed a supposition based on fig. 1 that the modes with lowest frequencies rotate in the direction of the electron diamagnetic velocity, in contrast to the modes with higher frequencies. Figure 2 demonstrates this for the modes with the same poloidal mode number $(|m|=5)$ in the discharge \#39029.

In order to see whether Drift-Sound Eigenmodes (DSE) exist in the framework of our model, we consider the simplest case of $\mu=\nu=0$. Then the drift-Alfvén and driftsound continua are decoupled; nevertheless, the equations determine the structure of DSE modes due to finite $\epsilon_{B}^{(00)}$. We write eqs. (3) and (4) for $\nu_{\iota}=0$ as follows:

$$
S \zeta_{m n}=p_{1} \Phi_{m+\mu, n+\nu N}^{\prime}+p_{2} \Phi_{m+\mu, n+\nu N},
$$

$$
\begin{aligned}
& \frac{d}{d r} A \frac{d \Phi_{m+\mu, n+\nu N}}{d r} \\
& -\left[\frac{(m+\mu)^{2}}{r^{2}} A+k_{m+\mu, n+\nu N}\left(r k_{m+\mu, n+\nu N}^{\prime}\right)^{\prime}\right] \Phi_{m+\mu, n+\nu N} \\
& =\left(q_{1} \zeta_{m n}\right)^{\prime}+q_{2} \zeta_{m n},
\end{aligned}
$$

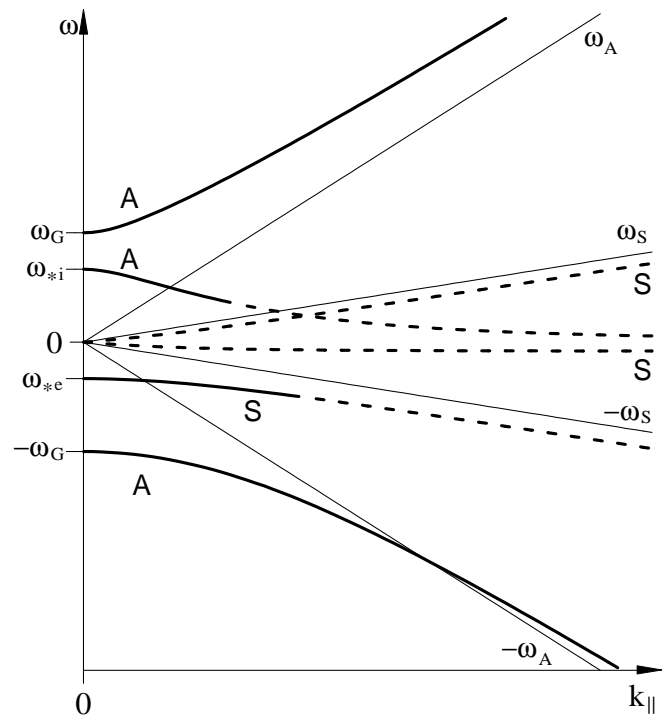

Fig. 1: Sketch of drift-Alfvén (labeled "A") and drift-sound (labeled "S") continuum branches for $\omega_{G}>\omega_{* i}>\left|\omega_{* e}\right|$. Notations: $\omega_{A}=k_{\|} v_{A}, \omega_{s}=k_{\|} c_{s}$, with $c_{s}$ the sound velocity. When $T_{e}=T_{i}$, only the parts of branches shown by bold lines are of interest because other branches lie in the region where $\omega \sim k_{\|} v_{\mathrm{th}, i}$; thus, the corresponding modes are strongly damped.

where the coefficients can be easily determined by comparing eqs. (7) and (8) with eqs. (3) and (4). We consider eqs. (7) and (8) in the vicinity of an extremum of the drift-sound continuum. Following an approach used in ref. [22] to study Alfvén eigenmodes, we obtain the following Schrödinger-type equation for $\mu=\nu=0$ :

$$
\frac{d^{2} \Psi}{d x^{2}}+[E-U(x)] \Psi=0,
$$

where $\Psi=\sqrt{A} \Phi, x=\left(r-r_{0}\right) / \Delta, r_{0}$ the radius where the continuum defined by $S\left(r_{0}, \omega\right)=0$ has an extremum, 

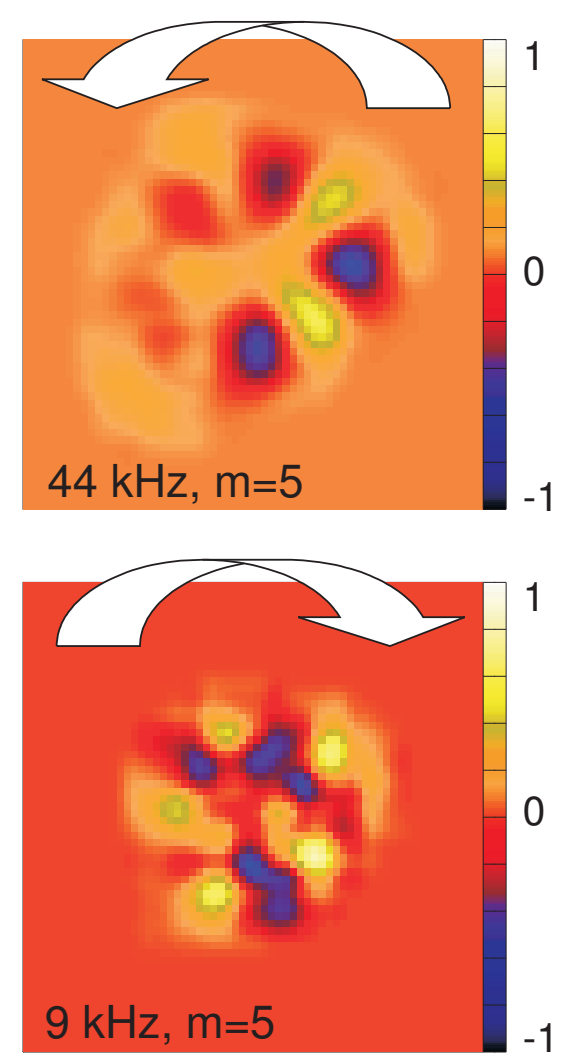

Fig. 2: (Color online) Tomographical reconstruction of soft Xray data in the W7-AS discharge \#39029. The high-frequency mode (GAE [3]) rotates in the ion diamagnetic direction, whereas the low-frequency mode rotates in the electron diamagnetic direction.

$\Delta^{2}=2 S /\left.S^{\prime \prime}\right|_{r_{0}}$, the energy $(E)$ and the potential $(U(x))$ are defined by

$$
\begin{gathered}
E=-\left[\frac{m^{2}}{r^{2}}+\frac{k_{m n}\left(r k_{m n}^{\prime}\right)^{\prime}}{A}+\frac{A^{\prime \prime}}{2 A}-\left(\frac{A^{\prime}}{2 A}\right)^{2}\right] \Delta^{2}, \\
U(x)=\frac{2 p_{2} q_{2}}{A S^{\prime \prime}} \frac{1}{1+x^{2}},
\end{gathered}
$$

where all the magnitudes are taken at the point $r_{0}$. One can see that $A<0$ (at least, when $\omega^{2}<\omega_{G}^{2}$ or $\omega^{2}<k_{\|}^{2} v_{A}^{2}$ ), $p_{2} q_{2}>0$ and $S_{\omega} \equiv \partial S / \partial \omega>0$ for $\omega<0 ; S^{\prime \prime} S_{\omega}>0$ at the maximum of the continuum and $S^{\prime \prime} S_{\omega}<0$ at the minimum. Therefore, there is a potential well at the maximum, and a hill at the minimum. The energy is typically negative (the first term dominates in eq. (10), and $\Delta^{2}>0$ for discrete modes). We conclude from here that discrete DSE modes exist when the continuum has a maximum (for $\omega<0$ ).

The conditions of existence of DSE with $\mu \neq \nu \neq 0$ and Drift-Alfvén Eigenmodes (DAE) can be investigated in a similar way. Note that, as follows from eq. (7), the longitudinal / transversal perturbations dominate when the mode frequency lies close to the $S=0 / A=0$ continuum branch, which justifies the names DSE and DAE.

Neglecting the mode coupling, we obtain the following Schrödinger-type equation for DAE modes:

$$
\frac{d^{2} \Psi}{d r^{2}}+\left[E_{1}-U_{1}(r)\right] \Psi=0,
$$

where $E_{1}=0$ and $U_{1}(r)=-E / \Delta^{2}$. A condition of the existence of discrete eigenmodes (not necessary well localized) is $U_{1}(r)<0$ in some region, which provides the presence of two "turning points". Discrete eigenmodes exist even when $U_{1}(r)<0$ in the whole plasma cross section provided that $\Psi(a)=0$, with $a$ the plasma radius [3].

Considering the case of $\mu \neq 0$, we take into account effects of the finite ion Larmor radius (FLR) by adding a fourth-derivative term, $\alpha r d^{4} \Phi_{m+\mu, n+\nu N} / d r^{4}$, to the lefthand side of eq. (8), as mentioned at the end of the previous section. Eliminating $\zeta_{m n}$ from eqs. (7) and (8), we obtain the following equation for drift-Alfvén and driftsound eigenmodes:

$$
\begin{aligned}
& \frac{d}{d r} F \frac{d \Phi_{m+\mu, n+\nu N}}{d r}-\left[\frac{(m+\mu)^{2}}{r^{2}} F-g\right] \Phi_{m+\mu, n+\nu N} \\
& +\alpha r \frac{d^{4} \Phi_{m+\mu, n+\nu N}}{d r^{4}}=0
\end{aligned}
$$

where $F=A-p_{1} q_{1} / S, g=-k_{m+\mu, n+\nu N}\left(r k_{m+\mu, n+\nu N}^{\prime}\right)^{\prime}-$ $\left(q_{1} p_{2} / S\right)^{\prime}$. Note that in the ideal limit case $(\alpha=0)$ eq. (13) possesses a continuous spectrum described by the equation $F=0\left(A S=p_{1} q_{1}\right)$. We approximate $F(r)$ as $F=F_{\omega}(\omega-$ $\left.\omega_{0}\right)\left(1+x^{2}\right)$, where $x=\left(r-r_{0}\right) / \Delta, \Delta^{2}=2 F_{\omega}\left(\omega-\omega_{0}\right) / F^{\prime \prime}$, $F_{\omega}$ and $F^{\prime \prime}$ are taken at a point $\left(r_{0}, \omega_{0}\right)$ where the continuum has an extremum. Assuming the rest of coefficients to be constant, we perform the Fourier transformation: $\Phi_{m+\mu, n+\nu N}(x)=\int d p \exp (i p x) \hat{\Phi}(p)$. The obtained equation can be reduced to the Schrödinger equation (9), in which $\Psi=\left(1+\bar{p}^{2}\right)^{1 / 2} \hat{\Phi}, \bar{p}=p / \mathcal{M}^{1 / 2}$ plays the role of $x$, $E=-\mathcal{M}, \mathcal{M}=\Delta^{2}(m+\mu)^{2} / r_{0}^{2}$,

$$
U(\bar{p})=\frac{1}{\left(1+\bar{p}^{2}\right)^{2}}-\frac{\bar{g}}{1+\bar{p}^{2}}-\frac{2(m+\mu)^{4} \alpha}{r_{0}^{3} F^{\prime \prime}} \frac{\bar{p}^{4}}{1+\bar{p}^{2}},
$$

where $\bar{g}=2 g / F^{\prime \prime}$. In the ideal case $(\alpha=0)$, eq. (9) with $U$ given by eq. (14) possesses a discrete spectrum when $\bar{g}>1 / 4$ [23]. The effect of the FLR term is determined by the sign of $F^{\prime \prime}$. When $F^{\prime \prime}<0$ and $\alpha>0$, FLR produces new modes in the continuum of the ideal equation (even when $\bar{g}<1 / 4$ and ideal modes are absent); we refer to them as Kinetic Drift-Alfvén Eigenmodes (KDAE) and Kinetic Drift-Sound Eigenmodes (KDSE). In the contrary case, $F^{\prime \prime}>0$, FLR results in radiative damping of ideal modes. Typically, KDSEs exist below the maximum of the continuum (because $F_{\omega}<0$ ). In the case of $\omega_{G}>\omega_{* i}$, KDAEs exist below the maximum when either $0<\omega<$ $\omega_{* i}$ or $\omega<0$ and $|\omega|>\omega_{G}$; they exist above the minimum for $\omega>\omega_{G}$ (like kinetic GAEs [15]).

A numerical modelling of LF instabilities in the W7-AS discharges \#39029 and \#40173 was carried out. Earlier 


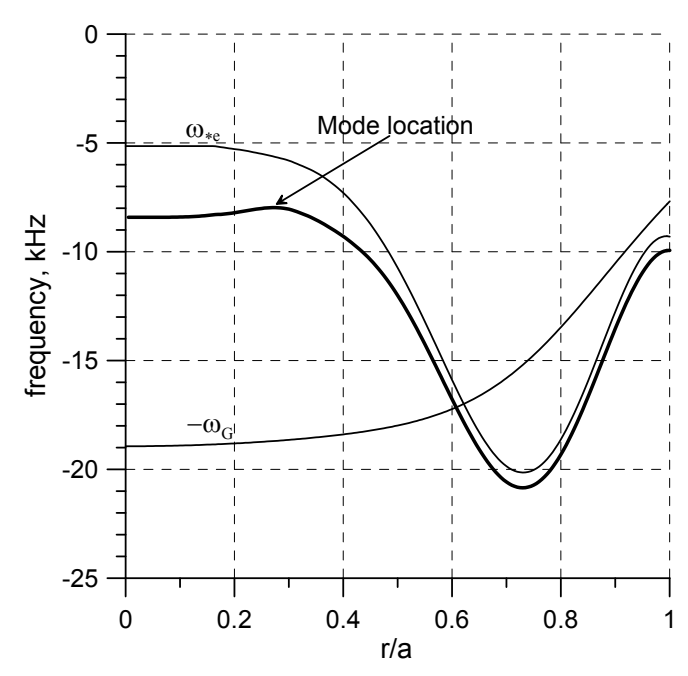

Fig. 3: A drift-sound continuum branch with $m=-5, n=-2$ and the eigenmode location in the W7-AS discharge \#39029.

these discharges were analyzed in refs. [3,24], where some of the observed modes were identified as GAE and NGAE modes. However, the theory of the mentioned works failed to explain the existence of the modes with the lowest frequencies (9 kHz in the discharge \#39029 and $16 \mathrm{kHz}$ in the discharge \#40173). Now we can suggest an explanation: The code BOAS finds new modes. In the discharge \#39029, they are DSE and KDSE with the frequencies close to $9 \mathrm{kHz}$ and the mode numbers $m=-5, n=-2$ (see fig. 3), which means that the observed mode may be either "ideal" or kinetic. In the discharge \#40173, it is an $m=-3, n=-1$ DAE mode. This mode appears due to finite $\omega_{* i}$; its frequency lies near the lowest branch with $\omega>0$ in fig. 1. This implies that the mode disappears in the ideal MHD and, thus, its nature differs from that of BAE.

The found modes satisfy the condition $v_{\text {th }, i} \ll \omega / k_{\|} \ll$ $v_{\text {th }, e}$. They can be destabilized by injected beam ions through the resonance $\omega=\left(k_{m+\mu, n+\nu N} \pm \iota / R\right) v_{\|}^{\text {res }}$ due to the velocity anisotropy. One can see that a solution of this equation is $v_{\|}^{\text {res }} / v_{0}=0.63$ ( $v_{0}$ is the velocity of injected ions) in the discharge \#39029, which means that the drive can exceed damping when $0.63<\chi<0.77$, where $\chi$ the pitch angle of fast ions (this condition is obtained for the distribution function $f_{b} \propto \delta\left(\chi-\chi_{0}\right) / v^{3}$ with the use of results of ref. [25]). In addition, spatial inhomogeneity of the electrons may contribute to the destabilization of modes with $\omega<0$.

Summary and conclusions. - The existence of DSE / KDSE modes in toroidal plasmas is shown for the first time and a new kind of DAE modes (the modes with $\left.\omega \lesssim \omega_{* i}\right)$ is predicted. Modelling of particular discharges of the Wendelstein 7-AS stellarator seems to confirm the existence of these modes. The DAE modes observed in the considered W7-AS discharges rotate in the ion dia- magnetic direction, whereas the DSE modes rotate in the electron diamagnetic direction, which agrees with theory predictions. In contrast to the modes with $\omega<\omega_{G}$ theoretically proposed earlier (BAE, KBM, and BAAE $[7,12,13,26-29])$, the new modes are located near flux surfaces where the continuous spectrum has extrema, which are not necessarily rational flux surfaces. Note that the DSE modes with small $k_{\|}$are characterized by negligible ion Landau damping, which facilitates their destabilization; this contrasts to the properties of the BAAE modes, which are strongly damped in isothermic plasmas. The derived equations [eqs. (3) and (4)] based on two-fluid hydrodynamics, as well as the developed numerical code BOAS, are applicable to both tokamaks and stellarators. Moreover, they can be used for modelling wave phenomena with $\omega \sim \omega_{* i, e}$ in other laboratory devices and space plasmas with inhomogeneous equilibrium magnetic field (in which case finite $\epsilon_{B}^{(00)}$ may play an important role).

The analysis carried out gives a new evidence that plasma compressibility, the electron and ion diamagnetic frequencies, and other factors neglected in ideal MHD may play an important role in physics of low-frequency instabilities in tokamaks and stellarators.

$$
* * *
$$

The work is carried out within the Project No. 4588 of the Science and Technology Center in Ukraine. One of the authors (Ya.K.) also acknowledges support by the International Bureau of the Federal Ministry of Education and Research (BMBF) at DLR in the frame of the CoOperation Project WTZ-UKR 06/005. The authors thank V. S. Marchenko for a useful discussion.

\section{REFERENCES}

[1] Heidbrink W. W. et al., Phys. Rev. Lett., 71 (1993) 855.

[2] Fasoli A. et al., Plasma Phys. Control. Fusion, 44 (2002) B159.

[3] Kolesnichenko Ya. I., Lutsenko V. V., Weller A. et al., Phys. Plasmas, 14 (2007) 102504.

[4] Sharapov S. et al., Phys. Plasmas, 9 (2002) 2027.

[5] Fu G. Y. and Berk H., Phys. Plasmas, 13 (2006) 052502.

[6] Kolesnichenko Ya. I., Yakovenko Yu. V., Weller A. et al., Phys. Rev. Lett., 94 (2005) 165004.

[7] Gorelenkov N. N. et al., Phys. Lett. A, 370 (2007) 70.

[8] Brüdgam M. et al., in Proceedings of the 10th IAEA TM on Energetic Particles in Magnetic Confinement Systems, 8-10 October 200\%, Kloster Seeon, Germany (IAEA, Vienna) 2008, report P-21.

[9] NAZIKIAN R. et al., Ibid., report OT-5.

[10] Winsor N., Johnson J. L. and Dawson J. M., Phys. Fluids, 11 (1968) 2448.

[11] Weller A. et al., Phys. Plasmas, 8 (2001) 931.

[12] Tsai S. T. and Chen L., Phys. Fluids B, 5 (1993) 3284.

[13] Zonca F., Chen L. and Santoro R. A., Plasma Phys. Control. Fusion, 38 (1996) 2011.

[14] Connor J. W., Hastie R. J., and Taylor J. B., Phys. Rev. Lett., 40 (1978) 396. 
[15] Mahajan S. M., Phys. Fluids, 27 (1984) 2238.

[16] Zonca F. et al., Phys. Plasmas, 9 (2002) 4939.

[17] Ramos J. J., Phys. Plasmas, 12 (2005) 052102.

[18] Ramos J. J., Phys. Plasmas, 12 (2005) 112301.

[19] Rosenbluth M. N. and Simon A., Phys. Fluids, 8 (1965) 1300.

[20] Hasegawa A. and Chen L., Phys. Rev. Lett., 35 (1975) 370.

[21] Zonca F., Chen L., Santoro R. A. and Dong J. Q., Plasma Phys. Control. Fusion, 40 (1998) 2009.

[22] Mahajan S. M., Ross D. W. and Chen G. L., Phys. Fluids, 26 (1983) 2195.

[23] Burdo O. S. et al., Plasma Phys. Control. Fusion, 36 (1994) 641.

[24] Kolesnichenko Ya. I., Lutsenko V. V., Weller A. et al., Ukrainian Journal of Physics, 48 (2008) 476.

[25] Belikov V. S., Kolesnichenko Ya. I. and Silivra O. A., Nucl. Fusion, 32 (1992) 312.

[26] Chu M. S. et al., Phys. Fluids B, 4 (1992) 3713.

[27] Turnbull A. D. et al., Phys. Fluids B, 5 (1993) 2546.

[28] Huysmans G. T. A., Kerner W., Borba D. et al., Phys. Plasmas, 2 (1995) 1605.

[29] Gorelenkov N. N. and Heidbrink W. W., Nucl. Fusion, 42 (2002) 150. 